\title{
VT catheter ablation and mapping system may decrease the rate of sudden cardiac death through VF/VT in patients with an implantable cardioverter defibrillator
}

\section{Authors: Emilia V. Goanta, P. S.Trasca, A. Ungureanu, O. Istratoaie, V. Georgescu, Luciana T. Rotaru. UMF Craiova/ University County Hospital Craiova,Romanian National Resuscitation Council}

\section{INTRODUCTION}

Patients with ventricular tachycardia (VT) and a history of myocardial infarction are at high risk of recurrent VT, or $\mathrm{VF}$, and sudden death. In patients with high risk of ventricular arrhythmia, intervention with an implantable cardioverter defibrillator (ICD) reduces rates of sudden cardiac death and total mortality compared with treatment with antiarrhythmic drugs. However, patients who receive ICD shocks have a decreased quality of life and increased mortality compared with patients who do not receive shocks, even if the shocks are inappropriate

\section{CASE REPORT}

A 65 years old man presented to the ED for palpitations, dizziness and multiple ICD shocks.

Patient had a background of inferior myocardial infarction occlusion in the middle segment and a coronary stent Xience 2,75/24mm; with good flow, TIMI 3 in the right coronary artery (two years ago), followed by an implantable dual chamber ICD (St. Jude Ellipse) for recurrent monomorphic VT (fig. la) 3 months after. He was on metoprolol and amiodarona since then.

At presentation - hemodynamically stable, sinus rhythm with SAP of $120-130 \mathrm{mmHg}$ (fig. Ib), with negative troponin levels, no biochemical or clinical signs of myocardial ischemia. During interrogation of the ICD 14 shocks were observed in the device memory, ineffective with proarrhythmic effect, with degeneration into VF (fig. 2a). Treatment with cordarone was discontinued and sotalol treatment was initiated for ineffective shocks. The fluoroscopic image of the ICD - fig 3.

Transthoracic echocardiogram showed akinetic inferior wall and an overall LVEF of $45 \%$ without further modifications compared to the previous examination

Control coronarography - a patent stent without other lesions.

An emergency ablation procedure was scheduled. After accesing the left ventricle with a three dimension mapping system, the voltage map was performed. A scar with area of $22 \mathrm{~cm}^{2}(10 \%$ of the left ventricle aria -fig $2 b$ ) was observed.

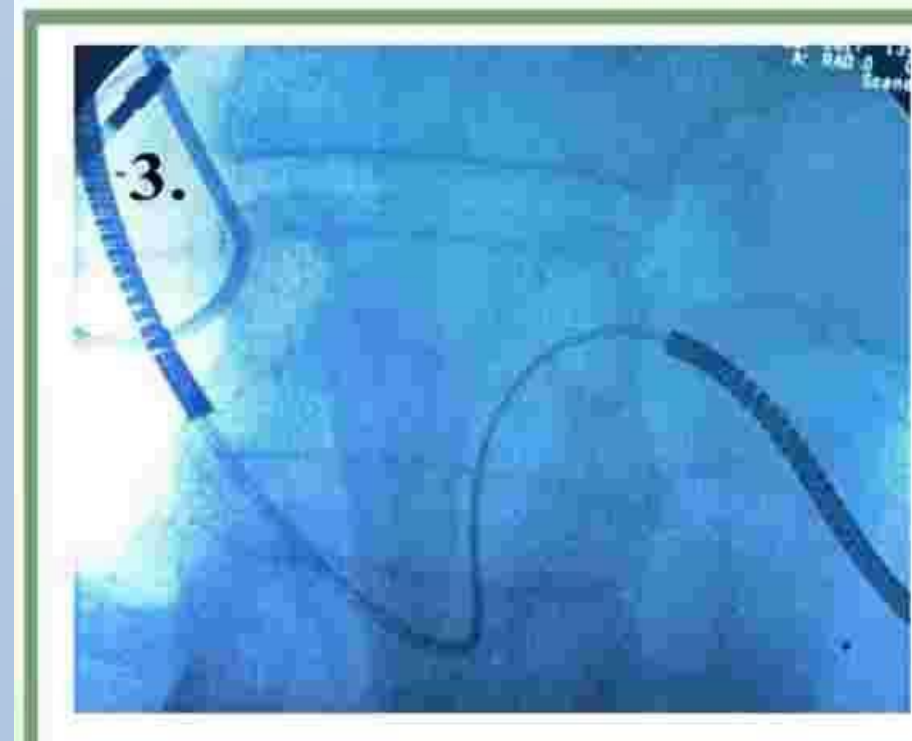

Antero-posterior projection of the ICD The fluoroscopic image of the ICD revealed the proximal coil at the right atrium level, device reprogramming was needed and proximal coil was excluded from the shock configur.ation
$1 a$
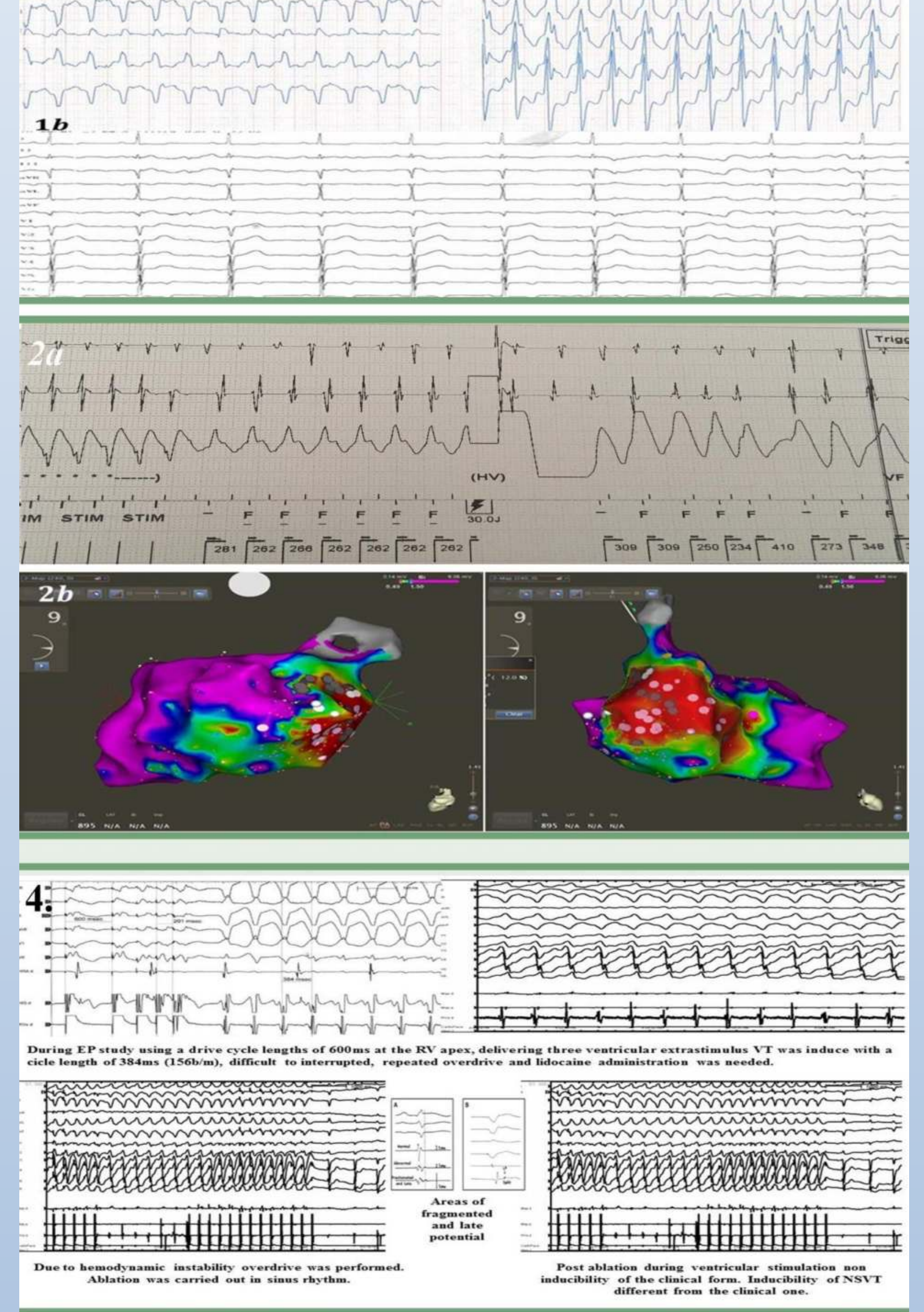

During electrophysiological study with PVC using a drive cycle lengths of $600 \mathrm{~ms}$ at the RV apex, delivering three ventricular extrastimulus VT was induce with a cicle length of $384 \mathrm{~ms}(156 \mathrm{~b} / \mathrm{m})$, difficult to interrupted, repeated overdrive and lidocaine administration was needed. Ablation was performed in sinus rhythm. We target the areas of fragmented and late potential, postablation without inducibility of clinical VT (fig 4). Follow-up at 6 months, 1 and 2 years after without any episoades of VT/VF or ICD shocks. 